\title{
Effectiveness of Rational Emotive Behavioural Group Counselling for Posttraumatic Stress Disorder in Orphan Children Found in Kechene Children's Home
}

\author{
Shimelis Keno Tulu \\ Department of Psychology, College of Social Sciences and Languages, Mekelle University, Mekelle, Ethiopia \\ Email address: \\ shimelis.keno@yahoo.com, shimeliskeno8@gmail.com

\section{To cite this article:} \\ Shimelis Keno Tulu. Effectiveness of Rational Emotive Behavioural Group Counselling for Posttraumatic Stress Disorder in Orphan \\ Children Found in Kechene Children's Home. American Journal of Applied Psychology. Vol. 3, No. 6, 2014, pp. 151-158. \\ doi: 10.11648/j.ajap.20140306.16
}

\begin{abstract}
In this study, a non-equivalent control group pre and post-tests of quasi-experimental research design was employed. The study was examined the effectiveness of Rational Emotive Behavioral Group Counseling for posttraumatic stress disorder in orphan children of Kechene Children's Home found in Addis Ababa, Ethiopia. Using the inclusion criteria, 60 participants aged 12 to 17 years old were purposefully selected and randomly assigned to the control and the treatment groups, with 30 participants in each group. The child posttraumatic stress symptoms scale was employed to measure the dependent variable before and after intervention. Participants in the treatment group were received 12 sessions of rational emotive behavioral group counseling for 4 successive weeks, 3 sessions per week; each session last for 1 hour. Results obtained from dependent t-test revealed that, after the intervention there was statistically significant reduction in the level of posttraumatic stress symptoms among participants in the treatment group $(\mathrm{df}=29, \mathrm{t}=2.245, \mathrm{p}<0.05)$ compared to participants in the control group. This result implies that, rational emotive behavioral group counseling is effective for the treatment of posttraumatic stress disorder in orphan children. Hence, it is recommendable to employ rational emotive behavioral group counseling in the intervention for children with posttraumatic stress disorder.
\end{abstract}

Keywords: Orphan Children, Posttraumatic Stress Symptoms, Posttraumatic Stress Disorder

\section{Introduction}

Since the emergence of HIV/AIDS, there was a dramatic increase in the number of orphan children in many countries around the world. The rapid increment in the number of orphan children in many countries has already reached the crisis level and nowadays the issue has become the major area of concern than any other time in the past [1].The HIV/AIDS epidemic alone has left behind 15 million orphan children worldwide [2]. It was also estimated that by 2010 the number of children orphaned by HIV/AIDS on the world will be 25 million [3].

In addition to HIV/AIDS, deaths of parents due to different causes have left 143 million orphan children across 93 countries of sub-Saharan Africa, Asia and Latin America [1]. It was estimated that more than 106 million children worldwide are projected to loss one or both parents from all causes by 2010 [4]. The present figure is beyond what was estimated for the fact that, over 145 million children worldwide are categorized as either single orphan or double orphan losing their fathers, mothers or both parents due to all causes [5].

The situation of orphan children in Africa is more shocking as over 14 million of African children were already orphaned and it is estimated that over 20 million additional children will be orphaned by the end of 2010 [6]. Currently, Sub-Saharan Africa alone is home to more than 12 million orphan children and it is estimated that this number will jump to over 16 million orphan children by the end of 2010. It was also estimated that more than 18.4 million children in SubSaharan Africa will be orphaned by 2010 losing their parents mostly due to HIV/AIDS [2].

Like other Sub-Saharan African countries, Ethiopia is characterized by large number of orphan children. For instance; in 2002 Ethiopia was home to over 77,000 orphan children holding the second rank next to Zimbabwe in sub- 
Saharan Africa [7]. Moreover, 18\% of all Ethiopian families are caring for one orphan and 5.5 million children which account for $6 \%$ of the total population are categorized as orphans. Similarly, there is an estimation that between 800,000 and 1.2 million children found in Ethiopia have been orphaned only due to HIV/AIDS and related factors [4]. It was also reported that around 4.6 million children who are roughly $10 \%$ of all children population in Ethiopia are categorized as orphans [3].

What is headache to many developing countries like Ethiopia is not only how to reduce the increasing number of orphan children but also how to deal with different psychological problems of these orphans. The findings of different studies indicated that orphan children are highly vulnerable to different psychological disorders such as PTSD, depression, anxiety disorder and low self esteem.

Therefore, orphan children are highly in need of effective counseling to cope up with their problems [4].

Different research findings also indicated that majority of orphan children are the victims of different traumatic events such as rape, sexual abuse, parental death and sudden accidents and more likely to develop PTSD [2]. PTSD is characterized by symptoms such as sleep disturbances, nightmares, irritability and flashbacks that can be experienced after exposed to life-traumas [8].

Orphan children are more vulnerable to different lifethreatening events under different situations. But, it is difficult for these orphan children to get effective counseling and cares following the experiencing of traumas. Hence they are at high risk of developing PTSD [9]. Majority of orphan children are more likely to develop PTSD following the experience of traumatic event [10]. Under various conditions, orphan children are highly exposed to multiple stressors that make their life more terrible and there is no or little formal counseling service available for them at a time they are in need of that [11].

Moreover, orphan children may have witnessed the unexpected death of their parent, parental conflicts or/and hostile divorce. Under such life intimidating circumstance, orphan children have high possibility to develop PTSD more than other group of children [2]. It was found that female orphans who have experienced rape, domestic violence and sexual abuse are highly vulnerable to PTSD than male orphans [9]. For instance; the result of one study revealed that, among 100 orphan children who have experienced sexual abuse and domestic violence, majority of them (72\%) were found to have PTSD [12]. Generally, it can be said that majority of orphan children exposed to traumatic event develop PTSD. Therefore, effective counseling service is very important for this group of children so that they cope up with their problems.

Rational Emotive Behavioral Therapy (REBT) is an eclectic, time limited, solution-focused and psycho educational counseling that basically focused on bringing long-lasting change. The basic tenet of REBT is that psychological disorders are caused by external situations and the belief of people about these situations [13].
REBT mainly deals with changing the clients' irrational thoughts to rational ones and allow the clients to develop realistic life goals [14]. The therapeutic approach of REBT is based on scientific, flexible and simple principles that can be used effectively for the intervention of various psychological disorders like Post-Traumatic Stress Disorder (PTSD) [15].

The therapeutic process of REBT is organized over an agreed number of sessions which is usually between a minimum of 8 sessions to a maximum of 16 sessions. Therefore, in REBT no fixed session to be followed by all counselors under all conditions. The treatment sessions can vary based on the nature and severity of the problems [16]. As REBT is brief and time limited, most of the time sessions are daily and last for an hour. For group counseling, many therapists preferred 12 sessions of REBT which is similar to other approach such as cognitive behavioral therapy [17].

Following intervention it is usually advisable to undertake a limited number of follow-up sessions to maintain the therapeutic gains and achieve more progress [15].

Rational Emotive Behavioral Group Counseling (REBGC) is a type of REBT that mainly help the clients in modifying their rigidity of thoughts and develop more flexible and workable philosophy of life [18]. Basically, REBGC is the application of major principles and basic therapeutic process of REBT in the context of group counseling [19]. REBGC is a time-limited and goal-oriented counseling that mostly focuses on bringing long-lasting change by altering irrational thoughts to more rational, flexible and beneficial ones[15]. REBGC is flexible and eclectic as it employs simpler psycho-educational approaches and widely used to treat different psychological disorders including such as irrational thought, behavioral disorders and PTSD [18].

REBGC is an active, goal oriented and time-limited counseling that basically focus on emotional, cognitive and behavioral aspects of the clients in solving the presenting dysfunctional thoughts [20]. In addition, REBGC focuses on the present difficulties and allow the clients to actively participate (like group discussions, and demonstrations) in identifying the underlying causes and setting counseling goals in collaboration with the therapist [14]. In the therapeutic process, REBGC emphasizes the clients' problems from cognitive, behavioral and emotional perspectives and emphasize on long-lasting change [21].

Therefore, this research is meant to assess the effectiveness of REBGC for Posttraumatic Stress Disorder in Orphan Children.

\section{Methods and Design of the Study}

This study is a non-equivalent control group pre test and post test quasi-experimental design. The design of quasiexperimental study can be presented by diagram as follows [22].

\begin{tabular}{lll}
\hline STUDY GROUPS & X \\
\hline Treatment group (TG) & $0_{1}$ & $0_{2}$ \\
Control group (CG) & $0_{3}$ & $0_{4}$ \\
\hline
\end{tabular}

Figure 1. Design of quasi-experimental study 
Where:

- Treatment Group: Was orphan children with PTSS as measured by CPSS and who received rational emotive behavioral group counseling.

- Control Group: Was orphan children with PostTraumatic Stress Symptoms (PTSS) as measured by Child Post-Traumatic Stress Scale (CPSS) and who didn't receive rational emotive behavioral group counseling (REBGC).

- $0_{1} \& 0_{3}$ : Refer to mean scores of dependent variable Post-Traumatic Stress Symptoms (PTSS) before treatment for the treatment and the control groups (CG).

- X: Refers to Rational Emotive Behavioral Group Counseling (REBGC) delivered to the participants in the treatment group (TG).

- $0_{2} \& 0_{4}$ : Refer to mean scores of dependent variable Post-Traumatic Stress Symptoms (PTSS) after treatment for the treatment and the control groups.

- The Line (........): Refers to the absence of participants' random selection.

\subsection{Research Site}

The study was conducted at Kechene Children Home $(\mathrm{KCH})$ that found in Gulalle sub-city of Addis Ababa City Administration. $\mathrm{KCH}$ is a local non-governmental organization aimed at providing various services such as accommodation, food, education, medical care and counseling for orphan children who have lost their parents due to different causes. $\mathrm{KCH}$ has one counselor and one social worker who are providing counseling services for orphans. Currently, KCH is providing cares for 290 orphan children. The ages of all children are 17 and less and almost all of orphan children in the institution are girls except few boys whose ages are 7 and less.

When orphan children are nearly to leave the institution at the age of $18 \mathrm{KCH}$ provide financial support that help them to be self supportive after they leave the institution. Most of orphan children in $\mathrm{KCH}$ are victims of different types of lifethreatening traumatic event and suffered from social as well as psychological problems. Majority of these orphans are the victims of domestic violence, abuses, rape and sudden parental death. These social and psychological problems combined with terrible institutional life lead majority of orphan children in $\mathrm{KCH}$ to various psychological problems especially PTSD.

\subsection{Population and Sampling}

The population for the study was all 290 orphan children. The screening test (pre-test) was first administered to 75 orphan children who were assumed to have PTSD. The screening test was scored and participants who met the inclusion criteria were selected. The inclusion criteria were orphan children who:

- Have PTSD as measured by CPSS and agree to participate in the study.

- Will stay in the institution till the administration of post-test.

- Are aged 12 to 17 years and didn't receive REBGC before.

- Have been exposed to traumatic event relatively prior to the study. Based on the inclusion criteria, 60 children were purposefully selected as the sample participants.

\subsection{Randomization}

Using the inclusion criteria 60 eligible participants aged 12 to 17 years were purposefully selected. All participants were female who were diagnosed with PTSD as measured by CPSS. First, pre-test (also used as the screening test) was administered to 75 participants who were assumed to have PTSD. During the completion of pre-test (screening test) all participants were requested to write their name on the first page. The result of the pre-test (screening test) was properly scored and 60 eligible participants who met the inclusion criteria were selected out of 75 participants who have completed the screening test. The remaining 15 participants who failed to meet the inclusion criteria were excluded.

Following the purposeful selection of 60 eligible participants, the following procedures were followed to randomly assign the 60 participants to the treatment and the control groups using lottery technique: firstly, 60 pieces of paper were prepared. Secondly, letters "TG" which stand for "Treatment Group" were written on the first 30 pieces of papers and letters "CG" which stands for "Control Group" were written on the remaining 30 pieces of paper. Thirdly, all 60 pieces of paper were rolled and mixed on the table and all 60 participants were asked to pick up only one piece of paper out of 60 . Finally, all participants were asked to open the rolled pieces of paper and 30 participants who were picked up letter "TG" were randomly assigned to the treatment group and the remaining 30 participants who were picked up letter "CG" were assigned to the control group.

\subsection{Variables of the Study}

The Child Posttraumatic Stress Symptoms Scale (CPSSS)

To measure the severity level of Post-Traumatic Stress Disorder (PTSD) in orphan children the Child PostTraumatic Stress Symptoms Scale (CPSSS) was employed. CPSSS is used to measure the severity level of PTSD in children whose age is range from 8 to 18 [23]. CPSS is an instrument that designed to access the severity of diagnostic and Statistical Manual of Mental Disorder ( $4^{\text {th }}$ edition); APA, 1994) PTSS in children experienced traumatic event. CPSSS, which is a child version of the Post-Traumatic Diagnostic Scale, has good validity to assess the severity of PTSS and diagnosis in adults exposed to various traumas [23] .The CPSSS has two parts; the first part contains 17 items each item containing one question for each of the 17 DSM-IV PTSS to determine the frequency of symptoms in the past month. The second part contains 7 additional items deals with specific areas of life [23]. CPSSS takes not more than 10 minutes to complete it as a self-report measure.

CPSSS has high internal consistency that ranges from .70 
to .80 for the total and the subscales symptom scores. The test-retest reliability of CPSSS was ranges from good to excellent with .84 for the total score, .85 for reexperiencing, .63 for avoidance and .76 for hyper-arousal. CPSSS has .89 alpha coefficients and the result obtained from item analysis shows the exclusion of any item doesn't increase the internal consistency.

The CPSS convergent validity of the total scale score was compared with the severity rating of Child PTSD Reaction Index and the Pearson Product-moment correlation coefficient was found to be .08. In addition, the CPSSS has lower correlation with the measures of depression and anxiety than the measure of Child PTSD Index indicating the discriminant validity of the CPSSS [24]. All these results indicate that, CPSSS is a proper tool to assess the severity level of PTSD in children exposed traumatic event.

The instruction to answer the CPSSS items is that, in the first part of CPSSS that contains 17 items each participant is requested to fill in the number that best describes how frequently that specific problem has bothered him/here during the last two weeks. The answers for these 17 items are on a Likert-type scale with 4 alternatives. Accordingly: $0=$ not at all, $1=$ once a week, $2=2$ to 4 times a week and $3=5$ or more times a week. Hence, the total scores range from 0 to 51 . In the second part of CPSSS that contains 7 items, each participant is asked to answer the extent to which the problems that are stated in the first part of CPSS have interfered with his/her specific areas of life. The 7 items are scored dichotomously as either present (1) and absent (0). Hence, the scores range from 0 to 7 with higher scores showing greater functional impairment.

Regarding the scoring procedures of CPSSS, each of 17 items in part one is rated on a scale that range from 0 to 3 . The 17 items give up the total scores that range from 0 to51. This score is proper for children ages 8 to 18 years and also provides both total scores and score for all three symptoms sub scales. The rest 7 items in part two scored on either present (1) or absent (0) and give up a total score that range from 0 to 7 . When the scores of all 24 items summed up $(51+$ 7) the total scores that range from $0-58$ is obtained.

The clinical cutoff score of $\geq 11$ was established in the original article of CPSS by examining the distribution of the total scale scores for children with maximum and minimum PTSS. But, a cutoff 15 is more preferred for determining PTSD [24]. Hence, the level scores PTSS for children with PTSD are determined as follows:

- Scores from 0 to 15 indicate the minimum levels of PTSD symptoms.

- Scores from16 to 24 indicate the mild levels of PTSD symptoms.

- Scores from 25 to 39 indicate the moderate levels of PTSD symptoms.

- Scores from 40 to 58 indicate the severe levels of PTSD symptoms.

The English version of the CPSS was first translated to Amharic language by the researcher. Then the backward translation of the tool was also made to avoid meaning difference between the original and the second translations. Finally, the accuracy and reliability of the translated instrument were revised by experts.

\subsection{Pilot Testing}

To determine the reliability of the CPSS the screening test was first administered to 50 individuals who were selected from the total population and 30 individuals found to have PTSD as measured by CPSS, hence purposefully selected for pilot testing. Second, pilot testing was conducted on 30 purposefully selected individuals. Finally, the responses of pilot testing were scored and the reliability of the CPSS was assessed by using Cronbach Alpha, which was found to be 0.76 for part one and 0.78 for part two indicating that the instrument is reliable. Then some minor modifications such as including understandable words in the sentences and changing the format of the word were done on the CPSS.

\subsection{Consideration of Ethical Issues}

The following ethical issues were considered in conducting this study:

- To all participants objectives of the study were made clear in advance using the language they understand and informed consents were obtained from all participants.

- How to collect data and when to start REBGC for the experimental group was decided as per the participants' agreement.

- Participants were informed about the possible potential discomfort they may experience in the course of the study and they were guaranteed about the confidentiality of all communicated information, and

- Participants were informed about their right to withdraw at any time of the study.

\subsection{Data Collection Procedures}

To carry out the present study the following consecutive steps were followed:

- First the researcher requested the collaboration of the $\mathrm{KCH}$ counselors in identifying participants for screening test (pre-test). The counselors were selected 75 participants who were assumed to have PTSS for screening test (pre-test).

- The researcher was first politely approached participants and introduced himself. All participants were clearly informed about the objectives of the study, the overall process to be done and the confidentiality of the communicated information. Then all 75 participants were requested to complete the screening test (pre-test) and during the administration of screening test participants were helped with the problems of understanding items.

- Based on the results of the screening test (pre-test) and others inclusion criteria only 60 participants were purposefully selected. The rest 15 participants who completed the screening test but not have PTSD as 
measured by the CPSS were excluded.

- Then, 60 participants were randomly assigned to the treatment and the controlled groups, each group with 30 participants using lottery technique. Based on their consent 30 participants in the treatment group were further divided into 2 sub-groups to create an appropriate counseling condition. Accordingly, 17 participants were willing to follow the intervention program during morning time, whereas the remaining 13 participants were welling to follow the treatment program during the afternoon time. As some of the participants in the treatment group were students school programs were also considered in assigning them into 2 sub treatment groups.

- After that, participants in the treatment group were encouraged to start and actively participate in 12 sessions of REBGC, 3 sessions per week (on Wednesday, Saturday and Sunday) for 4 successive weeks. REBT is so flexible and has no fixed number of sessions must be used by all therapists. Some literatures suggest that the total numbers of session in REBT vary between 5 to 30 sessions based on the nature and severity of the clients' problems. But, most of the time 8 to 16 sessions of REBT is preferred and with this range the number of sessions is determined reasonably [17]. For group counseling 12 sessions is preferred and sessions are normally daily and last for 1 hour [16].

- Participants in the treatment group were successfully followed 12 sessions of REBGC and post-test was administered for all participants in both the treatment and the control groups.

- Pre-test and post-test scores of the treatment group were compared to see any statistically significant change on the scores. In addition, post-test scores of the control group were also compared with the post-test scores of the treatment group to cross check if there is statistically significant difference due to the effectiveness of REBGC delivered to participants in the treatment group.

\subsection{Methods of Data Analysis}

The collected data was analyzed using the statistical package for social sciences (SPSS) version 15.0. Descriptive statistics, frequency distributions and percentage were used to analyze the demographic characteristics and the prevalence of PTSD. To compare the mean difference of the treatment and the control groups existed before and after treatment measures, dependent and independent t-test were used with alpha 0.05 levels of significance.

\section{Result}

\subsection{Demographic Characteristics of the Participants}

Data collected from participants were analyzed and presented below in line with the objectives of the study and available related literature findings.

Table 1. Demographic characteristics of participants in the treatment and control groups

\begin{tabular}{|c|c|c|c|c|c|}
\hline \multirow{2}{*}{ Characteristics } & & \multicolumn{2}{|c|}{ Control group } & \multicolumn{2}{|c|}{ Treatment group } \\
\hline & & Mean & SD & Mean & SD \\
\hline \multirow[t]{3}{*}{ Age } & $12-17$ & 15.60 & 1.65 & 15.76 & 1.47 \\
\hline & & Frequency & Percentage & Frequency & Percentage \\
\hline & Male & - & - & - & - \\
\hline \multirow[t]{3}{*}{ Sex } & Female & 30 & 100 & 30 & 100 \\
\hline & Total & 30 & 100 & 30 & 100 \\
\hline & Illiterate & 4 & 13.33 & 7 & 23.33 \\
\hline \multirow[t]{3}{*}{ Level } & Secondary & 4 & 13.33 & 3 & 10 \\
\hline & Total & 30 & 100 & 30 & 100 \\
\hline & $<1$ Month & 4 & 13.33 & 2 & 6.67 \\
\hline \multirow{4}{*}{$\begin{array}{l}\text { Time stayed in } \\
\text { the institution }\end{array}$} & 1 to 2 Months & 10 & 33.33 & 12 & 40 \\
\hline & 3 to 4 Months & 14 & 46.67 & 13 & 43.33 \\
\hline & 5 to 6 Months & 2 & 6.67 & 3 & 20 \\
\hline & Total & 30 & 100 & 30 & 100 \\
\hline \multirow[t]{3}{*}{ Counselling received } & No & 30 & 100 & 30 & 100 \\
\hline & Total & 30 & 100 & 30 & 100 \\
\hline & Yes & - & - & - & - \\
\hline \multirow[t]{2}{*}{ Received REBGC } & No & 30 & 100 & 30 & 100 \\
\hline & Total & 30 & 100 & 30 & 100 \\
\hline
\end{tabular}

Table 1, above indicates, all participants $(100 \%)$ in both groups were females. Regarding the educational background, majority of participants in the control group (73.34\%) and treatment group (66.67\%) were elementary school level. In addition, $46.67 \%$ participants in the control group and $43.33 \%$ of participants in the treatment group stayed in the institution for 3 to 4 months. When participants were asked if they had ever received any type of counseling, all participants (100\%) in both groups responded "NO". Moreover, participants were also asked if they had ever received REBGC before and all participants $(100 \%)$ in the both groups responded "NO". The above analysis evidently indicated that there were fair distributions of participants in the control and the treatment groups. 


\subsection{Posttraumatic Stress Symptoms in Orphan Children}

Table 2. PTSS level of the treatment group before and after treatment $(N=30)$

\begin{tabular}{lcccc}
\hline \multirow{2}{*}{ Level of PTSS } & \multicolumn{2}{c}{ Before treatment } & \multicolumn{2}{c}{ After treatment } \\
\cline { 2 - 5 } & Frequency & Percentage & Frequency & Percentage \\
\hline Minimum(0 to 15) & - & - & 2 & 6.67 \\
Mild(16 to 24) & 10 & 33.33 & 15 & 50 \\
Moderate(25 to 39) & 15 & 50 & 12 & 40 \\
Severe(40 to 58) & 5 & 16.67 & 1 & 3.33 \\
Total & 30 & 100 & 30 & 100 \\
\hline
\end{tabular}

Table 2, above shows that, majority of participants $(66.67 \%)$ in the treatment group showed higher level ( moderate or severe levels) of PTSS before the treatment, whereas after the treatment majority of the participants in the treatment group (56.67\%) showed lower level (mild or minimum levels) of PTSS as measured by the CPSS.

Table 3. PTSS level of the Control Group during the first test and the second test $(N=30)$

\begin{tabular}{lcccc}
\hline \multirow{2}{*}{ level of PTSS } & \multicolumn{2}{c}{ During the first test } & \multicolumn{2}{c}{ During the second test } \\
\cline { 2 - 5 } & Frequency & Percentage & Frequency & Percentage \\
\hline Minimum(0 to 15) & 3 & 10 & 2 & 6.67 \\
Mild(16 to 24) & 10 & 33.33 & 17 & 26.67 \\
Moderate(25 to 39) & 14 & 46.67 & 3 & 56.66 \\
Severe(40 to 58) & 3 & 10 & 30 & 10 \\
Total & 30 & 100 & 100 \\
\hline
\end{tabular}

As shown in table 3, majority of participants in the control group $(56.67 \%)$ during the first test and $(66.66 \%)$ during the second test have found to have higher level (moderate or severe levels) of PTSS as measured by the CPSS.

\subsection{Analysis of Dependent and Independent t-test}

Table 4. Dependent t-test of the mean PTSS scores of participants in the treatment group before and after treatment $(N=30)$

\begin{tabular}{lcccc}
\hline \multirow{2}{*}{ Treatment Group (TG) } & \multicolumn{4}{c}{ Posttraumatic Stress Symptoms Scores } \\
\cline { 2 - 5 } & Mean & SD & t-value & Significance \\
\hline Before treatment(pre-test) & 27.80 & 8.54 & & \\
After treatment(post-test) & 23.77 & 3.09 & 2.245 & $.033^{*}$ \\
Paired differences & 4.03 & 9.84 & & \\
\hline
\end{tabular}

$* \rightarrow$ Statistically significant, $\mathrm{p}<.05$

As indicated in table 4, the post test mean Post-Traumatic Stress Symptoms (PTSS) scores decreased to 23.77. A 2tailed t-test for statistically significant difference between the means revealed that the difference between the pre-test and post-test scores was statistically significant at 0.05 level of significance $(\mathrm{df}=29, \mathrm{t}=2.245)$. This finding imply that the provision of REBGC for participants in the treatment group has brought statistically significant reduction in the level of PTSS as measured by the CPSS from pre to post intervention.

Table 5. Dependent t-test of the mean PTSS scores of the control group during the first test and the second test $(N=30)$

\begin{tabular}{lcccc}
\hline \multirow{2}{*}{ Control group (CG) } & \multicolumn{4}{c}{ Posttraumatic stress symptoms score } \\
\cline { 2 - 5 } & Mean & SD & t-Value & Significance \\
\hline $\begin{array}{l}\text { During the first test } \\
\text { (pre-test) }\end{array}$ & 26.63 & 4.96 & & \\
$\begin{array}{l}\text { During the second test } \\
\text { (post-test) }\end{array}$ & 28.93 & 12.34 & -.912 & $.369^{\text {ns }}$ \\
Paired differences & -2.30 & 13.80 & & \\
\hline
\end{tabular}

$\mathrm{ns} \rightarrow$ Not statistically significant, $\mathrm{P}>.05$

From table 5, the mean difference in PTSS scores was 2.30. The result of dependent t-test revealed that there was no statistically significant difference between the first and the second tests mean of PTSS scores $(\mathrm{df}=29, \mathrm{t}=-.912)$. The slight increment observed in mean scores between the first and the second tests in the control group is ascribable to probability.

Table 6. Independent t-test of the mean PTSS scores of the control and treatment Groups before and after the treatment was given for participants in the treatment group

\begin{tabular}{|c|c|c|c|c|c|}
\hline \multirow{2}{*}{ PTSS Scores } & \multicolumn{5}{|c|}{ Groups } \\
\hline & Control & Treatment & Mean difference & t-value & Significance \\
\hline Before treatment (pre-test) & 26.63 & 27.80 & 1.17 & .647 & $.521^{\mathrm{ns}}$ \\
\hline After treatment (post-test) & 28.93 & 23.77 & -5.17 & -2.224 & .033 \\
\hline Mean differences & -2.30 & 4.03 & & & \\
\hline
\end{tabular}

$\mathrm{ns} \rightarrow$ not statistically significant, $\mathrm{p}>.05$ 
As presented in table 6, the provision of Rational Emotive Behavioral Group Counseling (REBGC) for participants in the treatment group has brought statistically significant reduction in the level of PTSS scores over the participants in the control group. That is, the mean scores of the treatment group decreased by 4.03 against -2.30 in the control group after 12 sessions of REBGC. A 2-tailed significance test for the equality of means indicated that there was a significant statistical difference between the control group and the treatment group during the post-test at 0.05 level of significance $(\mathrm{df}=58, \mathrm{t}=-2.224)$.

But, there was no statistically significant difference between the control group and the treatment group during the pre-test at 0.05 level of significance (2-tailed significance $=.521, \mathrm{t}=.647, \mathrm{df}=58)$. The implication of this finding is that participants in the treatment group had significantly minimized the level of PTSS when compared to participants in the control group as measured by the CPSS.

\section{Discussion}

The purpose of this study is to examine the effectiveness of REBGC for PTSD in orphan children. Thus, the next chapter deals with the discussion of results mentioned in the preceding chapter in line with previously done research findings.

\subsection{Posttraumatic Stress Disorder in Orphan Children}

As the findings of the present study indicated, before treatment $50 \%, 33.33 \%$ and $16.67 \%$ of participants in the treatment group have showed moderate, mild and severe levels of PTSS respectively. Similarly, 46.67\%, 33.33\%, 10\% and $10 \%$ of participants in the control group found to have moderate, mild, severe and minimum levels of PTSS respectively during the first test. This indicates that, before the intervention was given for participants in the treatment group $66.67 \%$ of participants in the treatment group have showed higher level that is, moderate or severe levels of PTSS. Similarly, $56.67 \%$ of participants in the control group have showed higher level that is, moderate or severe levels of PTSS as measured by the CPSS during the first test.

The findings of the present study are comparable with the previous research findings. For instance; [25] reported that, orphan children are five times more likely to experience higher levels of PTSS than non-orphan children. In addition, one study that was conducted on 150 orphan children of South Africa revealed similar findings with the present study as $50 \%$ of participants in the control group and $56 \%$ of participants in the treatment group have showed higher level (moderate or severe) levels of PTSS before the treatment as measured by the CPSS [26]. The findings of different studies also confirm that majority of orphan children are a victims of different traumatic events such as sexual abuse and sudden parental deaths. Hence these orphans are more likely to experience significantly higher levels of PTSS than other group of children [27].

After the provision of REBGC, participants in the treatment group have demonstrated low levels of the mean PTSS scores with statistical significance at $\mathrm{p}<0.05$. Moreover, when participants in the treatment group were compared with participants in the control group, participants in the treatment group have showed statistically significant reduction in the level of PTSS as measured by the CPSS. The result of the present study is comparable with the findings of some studies conducted previously. For example; in one study [28] found that clients who participated in REBT group counseling were showed a significant reduction in the symptoms of PTSD compared to clients in the comparison group. Similarly, the findings of one study conduct on children whose parents were divorced indicated that children treated by REBT group counseling with the group of 20 participants have showed statistically significant reduction in the symptoms of PTSD [29].

\section{Conclusions}

The following major conclusions were made based on the findings of this study:

- Before the treatment, majority of participants (66.67\%) in the treatment group and (56.67\%) in the control group have showed higher level that is, moderate or severe levels of PTSS as measured by the CPSS.

- Participants in the treatment group have showed a significant reduction in the level of PTSS from pre-topost-tests mean of PTSS scores after the completion of REBGC.

- Participants in the control group didn't show statistically significant reduction in the level of PTSS when the first and the second tests mean of PTSS scores was compared.

\section{Acknowledgements}

This Study is taken from my own MA thesis that I have conducted as fulfillment to my Master Degree in Counseling Psychology from Addis Ababa University. Hence, I would like to express my gratitude to Dr. Sintayehu Taddesse for his constructive comments from the conception to the end of this paper. Second, I would like to extend my gratitude to the counselors of Kechene Children's Home for all their unreserved collaboration.

Statement of the Competing Interest

The author has no competing interests

List of Abbreviations

- CG: Control Group

- CPSS: Child Post-Traumatic Stress Symptoms

- CPSSS: Child Post-Traumatic Stress Symptoms Scale

- KCH: Kechene Children's Home

- PTSD: Post-Traumatic Stress Disorder

- PTSS: Post-Traumatic Stress Symptoms

- REBGC: Rational Emotive Behavioral Group Counseling

- REBT: Rational Emotive Behavioral Therapy

- TG: Treatment Group 


\section{References}

[1] Osborn, K., Adolescents missing from programs for the world's orphan and vulnerable children: Advocates for youth. American Psychiatric Press, Washington, DC, 2007.

[2] Cluver, L., \& Gardner, F, "The mental health of children orphaned by HIV/AIDS" Journal of Child and Adolescent Mental Health, 19, 1-17. 2007.

[3] UNICEF, New estimation on orphan and vulnerable children: Responses to the national plan of action . New York, 2008.

[4] UNICEF, Africa's orphaned and vulnerable generations: Children affected by HIV/AIDS, Vintage Press, New York, 2006.

[5] Biemba, G., \& Beard, J, The scale, scope and impact of alternative care for orphan and vulnerable children in developing countries, Boston press, Boston, 2010, 12, 21-34, 2010

[6] USAID, Highly vulnerable childern: Causes, consequences and actions. Retrieved feburary 14, 2011, from htt://www.usaid.gov, 2007.

[7] Wubitu Hailu \& Solomon Gezahegne, Coping strategies of HIV/AIDS orphans children in wereda 5 and 11: Forum on Street Children- Ethiopia . Addis Ababa(Unpublished Material), 2009.

[8] UNICEF, Africa's orphaned and vulnerable generations: Children affected by HIV/AIDS, Vintage Press, New York, 2006.

[9] Murray, J., Ehlers, A., \& Mayou, R. A, "Dissociation and posttraumatic stress disoreder: Two prospective studies of road traffic accident survivors", British Journal of Psychiatry, 180, 3-36. 2002.

[10] Romanelli, H. L, "Mental health practices in child-welfare guidlines toolkit", Journal of Mental Health, 88, 1-7. 2009.

[11] Ahimed, A. Q, Two years follow up of orphans' competence, socio-emotional problems and posttraumatic stress symptoms, Dohuk Publishing Press, Dohuk, 2004.

[12] Zhao, W., \& Gelabert, J. M, "Nurturing care for China's orphaned children: National association for the education of young children", Journal of Young Children on the Web, 3(5),37-64. 2007.

[13] Rodriguez, N., Ryan, S. W., \& Foy, D. W, “Tension reduction and posttraumatic stress disorder: Open process group outcome among women with posttraumatic stress", Journal of Psychotherapy, 51(3), 379-398. 1992.

[14] Kumar, G. V, "Impact of rational emotive behavioral therapy on adolescents with conduct disorder", Journal of the Indian Academy of Applied Psychology, 35, 103-111.2009.

[15] Corey, G, Theory and practice ofcounseling and psychotherapy( $7^{\text {th }}$ ed. $)$. CA : Brooks/Cole. Belmont, 2009.
[16] Boni, M. D., \& Hurling, R, "Rational emotive behavioral therapy based automotived dialogue system for exercise behavioral change", Practicing Rational Emotive Behavioral Therapy, 2, 2-89. 2005.

[17] Ellis, A, Rational emotive behavioral therapy network. Retrieved March 5, 2011, from http://www.rebtnetwork.org/, 2006.

[18] Malkinson, R., \& Backx, W., "Rational emotive behavioral therapy successes and failures", Journal of Rational Emotive and Cognitive Behavioral Therapy, 24, (4), 22-54. 2007.

[19] Cluver, L, "Psychological distress amongest HIV/AIDS orphanage children in urban South Africa", Journal of Child Psychiatry and Psychology, 19, 2-19. 2000.

[20] Froggatt, W, Brief introduction to rational emotive behavioral therapy. Retrieved April 2, 2011, from htt://www.anapsts.co.uk/files/Brief\%20Introduction $\% 20$ to $\% 2$ OREBT, 2001.

[21] Bishop, F. M, "The basic of rational emotive behavioral therapy" National Training Conference (pp. 3-14). Phoenix: AZ, 3-14.

[22] Cohen, L., Manion, L., \& Morrison, K. Research Methods in Education $\left(5^{\text {th }}\right.$ ed.), Routledge Falmer, London, 2000.

[23] Lupu, V., \& Cosman, D, "Rational emotive behavioral therapy in the treatment of major depressive disorder", Journal of Clinical Psychology, 64, 728- 746. 2008.

[24] Foa, E. B., Riggs, D. S., Dancu, C. V., \& Rothbaum, B. O, "Reliability and validity of a brief instrument for assessing posttraumatic stress disorder", Journal of Traumatic Stress, 6(4), 459-473. 1997.

[25] Foa, E. B., Johnson, K. M., Feeny, N. C., \& Treadwell, K. R. H. "The child posttraumatic symptoms scale: A preliminary examination of its psychiometric properties", Journal of Clinical Child Psychology, 30, (3), 376-384. 2001.

[26] Bisson, J, "Posttraumatic stress disorder and mental health: Clinical cvidence", British Medical Journal, 1(2), 166-167. 2009.

[27] Seedat, S., \& Fincham, D. S, "Posttraumatic stress in HIV/AIDS orphaned children exposed to higher level of trauma: The prospective role of percieved social support", Journal of Traumatic Stress, 22, 2-15. 2009.

[28] Somasundaram, D, "Collective trauma in northern Sir-Lanka: A qualitative psychological and ecological study", International Journal of Mental Health Systems, 5, 17-44. 2007.

[29] Bernard, M, "The rational emotive behavior therapy therapist's pocket companion for working among women: A community sample". Journal of Interpersonal Violence, 7, 213. 2004.

[30] Bistamam, M. N., \& Nasir, R, "Application of rational emotive behavioral therapy in group counseling," European Journal of Social Sciences, 10, 14-54. 2009. 